\title{
FastTest Plugin: a new plugin to generate Moodle quiz XML files
}

\author{
Milagros Huerta ${ }^{1, *}$ and Manuel Alejandro Fernández-Ruiz²
}

1 Departamento de Ingeniería Mecánica y Diseño Industrial, Universidad de Cádiz; milagros.huerta@uca.es 2 Department of Industrial and Civil Engineering, University of Cádiz (UCA). Campus Bahía de Algeciras, Avda. Ramón Puyol, s/n. 11201 Algeciras (Cádiz), Spain. manuelalejandro.fernandez@uca.es.

* Correspondence author: milagros.huerta@uca.es

Featured Application: this new plugin helps Moodle's users to create big question banks.

\begin{abstract}
The use of Learning Management Systems (LMS) has had a rapid growth over the last decades. Great efforts have been recently made to assess the level of performance of the students online due to the COVID-19 pandemic. Faculty members with limited experience in the use of LMS such as Moodle, Edmodo, MOOC, Blackboard and Google Classroom can have some problems creating online tests. In this work, a new plugin for Moodle is presented: FastTest PlugIn. This plugin is based on a Microsoft ${ }^{\circledR}$ Excel spreadsheet and it can be used to export questions to Moodle. Users of FastTest PlugIn can create question pools in an easy and intuitive way. A literature review about plugins used to import/export questions in Moodle is carried out. Then, the characteristics of FastTest PlugIn are presented. At the end, the characteristics of the main plugins found in the literature are discussed and compared with the ones of FastTest PlugIn.
\end{abstract}

Keywords: content creation tools; plugin; Moodle; e-learning technologies; LMS; XML file; quiz.

\section{Introduction}

During the COVID-19 pandemic, all the teaching programs were switched to e-learning methods [1-3]. This was a big challenge for students and professors. In the literature there are numerous studies analyzing the impact of COVID-19 pandemic on teaching and learning [4-9]. Some universities were already familiar with the use of e-learning methods before the beginning of COVID-19 pandemic. In fact, e-learning methods have been used since the beginning of the XXI century [10-12]. According to Dahlstrom et al. [13] 99\% of institutions have Learning Management Systems (LMS) in place and 85\% of faculty members use it. For Spain, 85\% of the faculty members used almost daily LMS for synchronous or asynchronous online teaching during the lockdown provoked by COVID-19 pandemic [14]. However, $60 \%$ of faculty members did not have previous experience in online teaching [14]. The main difficulty of using e-learning methods reported by faculty were the lack of time $(62.1 \%)$ and their insufficient knowledge of the use of LMS $(29.6 \%)$.

Once online classes were established, the next step was to assess the level of performance of the students. At the beginning of COVID-19 pandemic, examinations were postponed. However, as pandemic continued, institutes of high education were forced to conduct online examination [15]. Online examination has its own advantages and disadvantages for institutions, faculty members and students [16-19]. It is considered to be more economical, environmental friendly (paper saving), and it has some other advantages such as auto-grading, student's authentication and effectiveness [20,21]. However, online assessment presents some disadvantages such as the lack of students' technological resources [22,23], network connectivity problems [24,25] and cheating detection $[26,27]$. 
One of the bigger challenges of online assessment is to ensure its credibility, security and validity. Prior to COVID-19 pandemic, cheating detection has been one of the main concerns of faculty members [28-31]. Recently some works have been published about this topic [32-35]. There is not a general agreement among faculty about which method of online examination is the most suitable to evaluate students' assessment $[17,36-38]$. In Boitshwarelo et al. [36] the use of online tests in assessing twenty-first century learning is studied. Online tests (also known as online quizzes) are a type of computer-assisted assessment where the deployment and grading are automated. The use of online tests has had a rapid growth over the last two decades due to the widespread implementation of LMS in higher education [39]. Gipps [40] pointed out the efficiency of online tests because of automated marking and feedback. According to Brady [41], online tests can assess a wide range of topics in a short duration. In addition, students show a positive attitude towards online testing [42,43]. However, it has to be pointed out that the effectiveness of online tests is increased when they are implemented together with other assessment methods [44].

Boitshwarelo et al. [36] proposed some principles for the design of online tests based on an extensive literature review. One of the most successful strategies to minimize cheating pointed out in [45] is the use of multiple versions of the test, randomizing question and response orders. In general, online tests can be composed by Multiple Choice Questions (MCQs), matching questions, true/false questions and short answer questions among others. The use of parameterized questions is a good option to develop individualized exercises. A parameterized question is a pattern of a question where some parameters (numbers and/or text) are randomly generated based on a specific set of replacement values. By doing so, each parameterized question is able to generate a large (or even unlimited) number of different questions. Consequently, individualized online tests can be generated with a reduced number of parameterized questions, which is very useful for large classes. Parameterized questions can also be set manually (which requires a lot of time and work) or using different tools provided into LMS.

LMS has had a great development over the last decades in STEM (Science, Technology, Engineering, and Mathematics) courses [46,47]. Currently, a high number of LMSs for academic/educational purposes can be found. LMSs such as Moodle, Edmodo, MOOC, and Google Classroom were highlighted as the most widely used and researched during 2015-2020 [48]. Comparisons between different LMSs can be found in the literature [49,50]. Moodle is the most popular open-source LMS according to a recent work [51]. This LMS is used worldwide due to its high rate of acceptance in the academic community [52]. The number of Moodle users has had an increasing trend in recent years, reaching 305 million at the beginning of 2022 [53]. As Moodle is an open-source LMS, it enables the creativity of its users. Moodle has a growing library of over 1900 plugins that are shared with the community [54]. The most used tools in Moodle are "quizzes" and "workshops" [46]. However, the steep learning curve required to transfer paper tests to Moodle platform and/or the creation of parameterized questions can be limitating for some faculty members with limited Moodle experience [55]. In order to solve this problem, Moodle allows the user to import questions in a variety of formats [56]. While this process requires some experience in import codes, there are some plugins that allow an easy conversion of text to Moodle questions. In fact, 145 of the 202 Moodle plugins of activity modules are Quiz activities, and 20 of them are question formats for import/export [57].

In this work, FastTest PlugIn [58] is presented as a new Moodle plugin to export questions. FastTest PlugIn is a Microsoft ${ }^{\circledR}$ Excel based plugin that allows to export MCQ, true/false questions, matching questions, short answer questions, essay questions, missing words questions, and Cloze questions in an easy and intuitive way. Hence, faculty members with limited Moodle experience can develop a question pool without requiring much time and effort. In Section 2, a literature review about plugins employed to import/export questions in Moodle is carried out. In addition, the most important plugins indicated are studied in greater detail. In Section 3, the new Moodle plugin called FastTest PlugIn is 
presented. In Section 4 a comparison between FastTest PlugIn and the plugins shown in Section 2 is carried out. Finally, the conclusions of the work are summarized in Section 5.

\section{Related Research}

In this section a literature review is carried out to point out previous works related with the research topic of this piece of work: plugins to import/export questions in Moodle. Afterwards, the characteristics of the main previous research works are discussed.

\subsection{Literature Review}

A literature review was conducted to identify Moodle plugins about the importation/exportation of questions. The formats used to import questions in Moodle are the following [59]:

- $\quad$ Aiken: a simple format for importing MCQs from a text file.

- $\quad$ Blackboard: a format for importing just text questions (.dat files) or question and associated media such as images, sounds... (.zip file).

- $\quad$ Embedded Answers (Cloze): a format for importing multi-type questions.

- $\quad$ Examview: a format for importing questions from ExamView4 XML files.

- GIFT: a Moodle proprietary format for importing MCQs, true/false, short answer, matching, missing word, numerical and essay questions via text file.

- Missing word: a format similar to GIFT for importing MCQs and short answer questions.

- Moodle XML: a Moodle proprietary format for importing questions via XML.

- WebCT: a format for importing short answer, multichoice single answer and multichoice multiple answers, calculated and essays saved in WebCT's text-based format.

Aiken, Cloze and Missing word formats are discarded because they can import only one type of questions. Blackboard, Examview and WebCT are also discarded because they are external platforms. Consequently, GIFT and Moodle XML are the import formats considered in this work. The first format enables the importation of a wide variety of type of questions with a simple coding. On the other hand, Moodle XML format enables the importation of all the types of questions that Moodle can create. Furthermore, it allows the importation of additional information beyond the proper question such as feedback and clues. The main drawback of Moodle XML format is that its programming code can be considered complex and non-intuitive for faculty members without any programming skills or knowledge.

Now that the questions' import formats are selected, a scoping literature review to identify Moodle's plugins to import questions in these formats was conducted. The search was done in the Moodle plugin directory, forums of the Moodle community, pieces of work presented in Moodle's conferences and other platforms. The key search terms used in the review were: import questions, quiz, Moodle, XML, GIFT, and plugin.

Considering the number of downloads, "Microsoft Word File Import/Export" is the most-downloaded Moodle plugin used to import/export questions collected in the "Question format" directory [60]. It was created in July 2012 and it has had almost 7000 downloads in the last year (an average of 20 downloads per day). The following plugin in the most-downloaded list is "H5P content types" [60], which was created in May 2020 and it has had 5000 downloads in the last year (an average of 14 downloads per day). This plugin is not considered in this piece of work because it enables the importation of various H5P content types into the Moodle question pool. The third position in the most-downloaded plugins list is "GIFT with medias format" [60]. This plugin was created in April 2013 and it has had little more than 900 downloads in the last year (an average of 3 downloads per day). The rest of plugins are used to import questions developed in other formats (such as CSV) and/or in other platforms than Moodle, and they are not considered in this piece of work. The plugins collected in the Moodle's directory "Utilities" has been also studied. The only plugin collected in this directory used to import questions is "FastTest Plugin". 
This new Moodle's plugin was added to the in Moodle's directory in December 2021, and has almost 230 downloads (an average of almost 8 downloads per day) [61].

Finally, some plugins to import/export questions in Moodle have been found in Moodle's forums. The most interesting plugins identified in this literature review has been described in Section 2.2.

There are some plugins for LaTeX users such as "Scientific WorkPlace" [62] and "Moodle package" [63]. The first one is limited to MCQs. "Moodle package" [63] allows the definition of a higher variety of question types. However, both plugins are not considered in this study because the users are required to have prior knowledge of LaTeX programming language.

\subsection{Main Moodle's plugins to import questions}

In this section, the characteristics of the main Moodle's plugins to import questions are indicated (operation, language, ease of use, types of questions, etc.). They have been classified based on the computing resources needed.

\subsubsection{Web-based plugins}

Web-based plugins can be used with any operating system and only an Internet connection is required.

\section{- VLEtools.com:}

This plugin allows the creation of quizzes and glossaries for Moodle [64]. It includes MCQs, true/false, short answer, essay, numerical, matching, order, description, and Cloze questions. The procedure for the creation of the questions is indicated in its webpage, with some illustrative examples. In VLEtools the question format is very similar to the GIFT one. An XML file is generated with all the questions ready to be imported into Moodle. VLEtools also allows the previsualization of the questions and provides information about possible errors and warnings made in their definition.

\section{- Moodle test creator:}

This web-based plugin allows the creation of a question pool for Moodle [65]. Moodle test creator is focused on managing a large number of questions, and can include MCQs, true/false, short answer, essay, and numerical questions. There are some specific rules for the definition of each type of question (in the webpage there are some illustrative examples). A GIFT file is generated with all the questions ready to be imported into Moodle. A warning message may appear if an error in the definition of the question is found.

\subsubsection{Spreadsheets plugins}

There are some plugins based on spreadsheets. For most of them, it is necessary to have installed Microsoft ${ }^{\circledR}$ Excel and they are developed using VBA programming language (macOS users can experience some problems). These plugins do not require an Internet connection to create the question pool.

\section{- CQ4M Generator:}

This plugin allows the creation of large question pools for Moodle based on Excel [66]. It includes MCQs, numerical, Cloze, and binomial distribution questions. Each type of question is in a different Excel file. Question data is directly introduced in the corresponding cells, with the exception of Cloze questions where the lines of code must be directly introduced. The spreadsheet is totally unlocked, so the user needs to be careful during the introduction of questions because important cells can be deleted unintentionally. In addition, if the question data is not introduced correctly there is no warning message. It is also the case when generating the XML file, so some errors can appear when the file is imported into Moodle. The language used in CQ4M Generator is English, but the message boxes of the macros are in Dutch. Excel functions can be used for calculated questions. Consequently, CQ4M Generator can create parameterized questions.

\section{- Moodle Cloze and GIFT Code Generator:}

Moodle Cloze and GIFT Code Generator is an Excel-based plugin that facilitates the question creation process generating lines of code ready to be imported into Moodle [55]. 
Different types of questions such as MCQs, true/false, missing word, short answer, essay and Cloze are supported. As the Excel spreadsheet is totally unlocked, the user has to introduce carefully the question data into the corresponding cells (but it is not necessary any line of code). Important cells can be deleted unintentionally and there is no warning message if this occurs. Cloze questions include Spelling Questions. Moodle Cloze and GIFT Code Generator generates a GIFT or XML file to be imported into Moodle.

\section{- Tool based on VBA for the generation of question pools in Moodle:}

This tool allows the generation of question pools for Moodle from an Excel spreadsheet [67]. The following question types are supported: MCQs, true/false and numerical questions. A tutorial to guide the user during the introduction of question data is provided. This tool also allows the introduction of images. Finally, an XML file is generated ready to be imported into Moodle.

\section{- $\quad$ FastTest PlugIn:}

This plugin is fully described in Section 4.

\subsubsection{Plugins developed in text processors}

In the literature there are some plugins developed in text processors such as $\mathrm{Mi}$ crosoft $^{\circledR}$ Word or OpenOffice Writer. As in the case of the plugins shown in Section 2.2.2, an Internet connection is not required to create question pools.

\section{- QT_Machining, QT_Cloze, QT_TrueFalse:}

In Hata et al. [68,69] different tools based on Word and Excel are presented. These plugins are not available online and all the information about them is in Japanese. For this reason, it is difficult to analyze their characteristics. However, it shows that the introduction of questions into Moodle is of major concern for faculty members all over the world.

\section{- $\quad$ Libre Gift:}

Libre Gift [70] allows the creation of questionnaires in GIFT and XML formats ready to be imported in Moodle. It is based on Open Office Writer (free software) and includes these types of questions: MCQ, true/false, missing word, short answer, matching, numerical, and Cloze questions. If question data is not correctly introduced, no warning message is displayed. Question pools are imported to Moodle in XML and GIFT formats.

\section{- Moodle2Word:}

Moodle2Wrod is a plugin based on Microsoft ${ }^{\circledR}$ Word that import questions (including images and equations) from structured tables in a .docx file. It also export questions to a Word file, allowing for round-trip editing [71]. The following types of questions are included in Moodle2Word: MCQs, all or nothing MCQs, true/false, missing word, short answer, matching, essay, description, and Cloze questions. It is necessary to have Moodle2Word installed in Moodle by an administrator. Moodle2Word's webpage provides sample templates of all the types of questions. The steps to be followed to enter the question data in the template is very clear, but the document is unlocked and some parts of it can be deleted unintentionally. The users see no warning message if the question data is not correctly introduced. Questions can be exported into XML and GIFT formats. Moodle2Word was developed in July 2012 and it is currently installed in almost 4000 Moodle's sites.

\subsubsection{Specialized software}

Finally, specialized software is also an option to create question pools to be imported into Moodle.

\section{- Question Machine:}

Question machine [72,73] is a free software that provides a user friendly Microsoft ${ }^{\circledR}$ Windows interface to create question pools ready to be imported into Moodle in XML format. It includes MCQs, true/false, short answer, numerical, matching, and Cloze questions. This software generates the XML file with all the questions, and the users can export all at once or attend to each type of question. If a question is incomplete, the users cannot 
save it. Images are easily managed and they are displayed on the screen ("what you see is what you get"). Question Machine is supported in Windows XP, Windows Vista and Windows 7.

\section{- Quiz Faber:}

Quiz Faber [74] is a free software for Windows for the creation of multimedia quizzes in HTML. The following questions are supported: MCQs, true/false, matching, and essay questions. In addition, these quizzes can be imported into Moodle (only for the question types supported by the latter). Images are easily managed and warning messages appear if the question data is incomplete.

\section{FastTest PlugIn}

A beta version of FastTest PlugIn $[58,75]$ was made available to the educational community in January 2021 through a YouTube channel [76]. Soon enough the need for an improved version of this plugin arose due to the evolution of the COVID-19 pandemic. Different versions of FastTest PlugIn were developed incorporating users' needs. Prior to its inclusion within Moodle's plugins directory, FastTest PlugIn had 645 downloads [77]. Then, in November 2021 FastTest PlugIn was included within Moodle's plugins directory and since then it has had 291 downloads (199 in January 2022). In this Section the main characteristics of this new Moodle's plugin are described.

\subsection{General operating principles}

The question data entry screens for the different types of questions have a uniform style in order to facilitate the introduction of the information (see Figure 1). In the data introduction, the user can move to the next cell by pressing the "tab" button. Orange cells must be filled (they must not be left blank). On the other hand, white cells are optional fields. Grey cells are used for the creation of parameterized questions. They contain the parameters of this type of questions. Grey cells appear on the "General Data" and "Form" screens and they can be activated or not. Warning cells are in yellow, and they show warning messages or they inform about a cell that is not correctly filled. However, they allow the user to continue the data introduction. Cells without borders contain plain text, and their content will be shown as it is in Moodle. For this reason, HTML syntax, images, and videos cannot be inserted in these cells. Furthermore, cells with double border can contain text in HTML, including images and videos links. All Fast Test PlugIn's screens have a link to the Moodle's Editor and show the version of the plugin. In addition, all the cells have their help option informing the users about the data expected.

The last version of FastTest PlugIn has implemented the Zoom effect. With this effect, the content of each cell can be viewed independently in a pop-up window (see Figure 2). In this pop-up window also appears the cell reference and some buttons to navigate between the different cells and to save the changes made. The Zoom effect is recommended for cells that contain long texts (like the example shown in Figure 2), because its visualization is difficult in the Excel spreadsheet.

Each screen has its drop-down menu, which is displayed by a right-click. Blue buttons activate different functions. All functions have keyboard shortcuts (that are indicated in the drop-down menu). In both "General Data" and "Form" screens there is a lateral menu that contains some special codes such as superscript, under script, bold, text color, etc.) in HTML syntax (see Figure 1). In addition, images available on the Internet can be introduced indicating its URL and its viewing dimensions. Finally, in the lateral menu, the variable data can be activated or deactivated. 


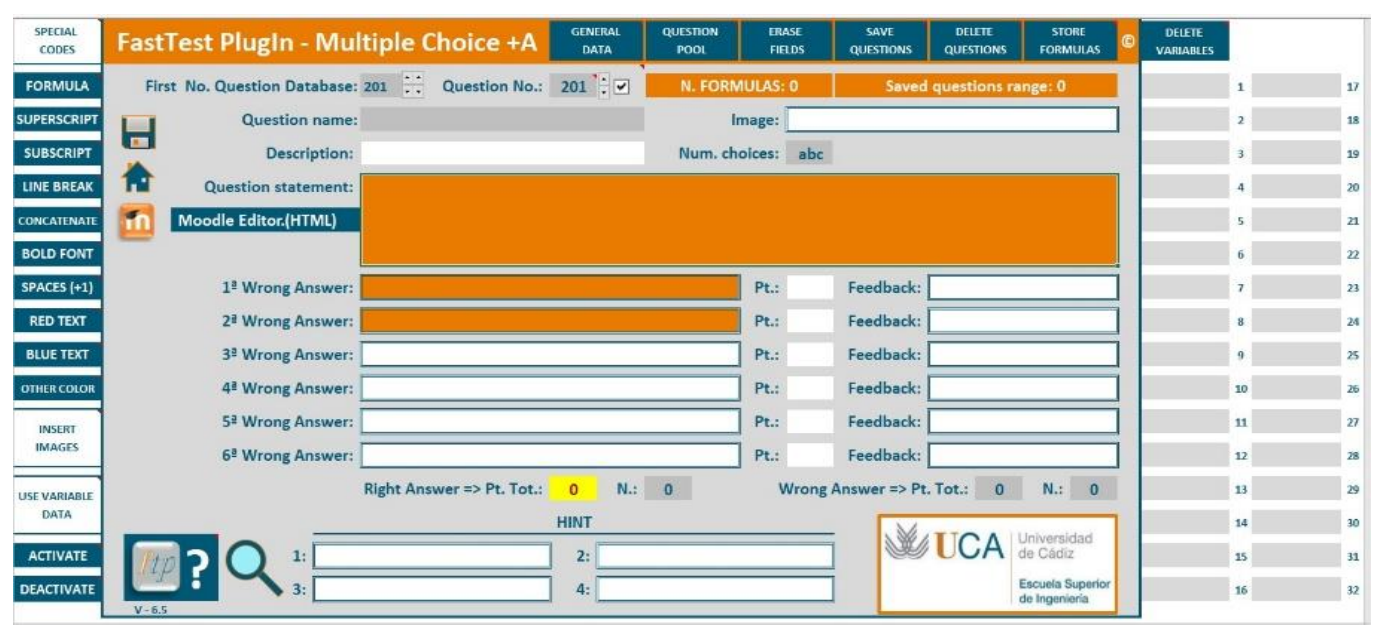

Figure 1. General question data entry screen.

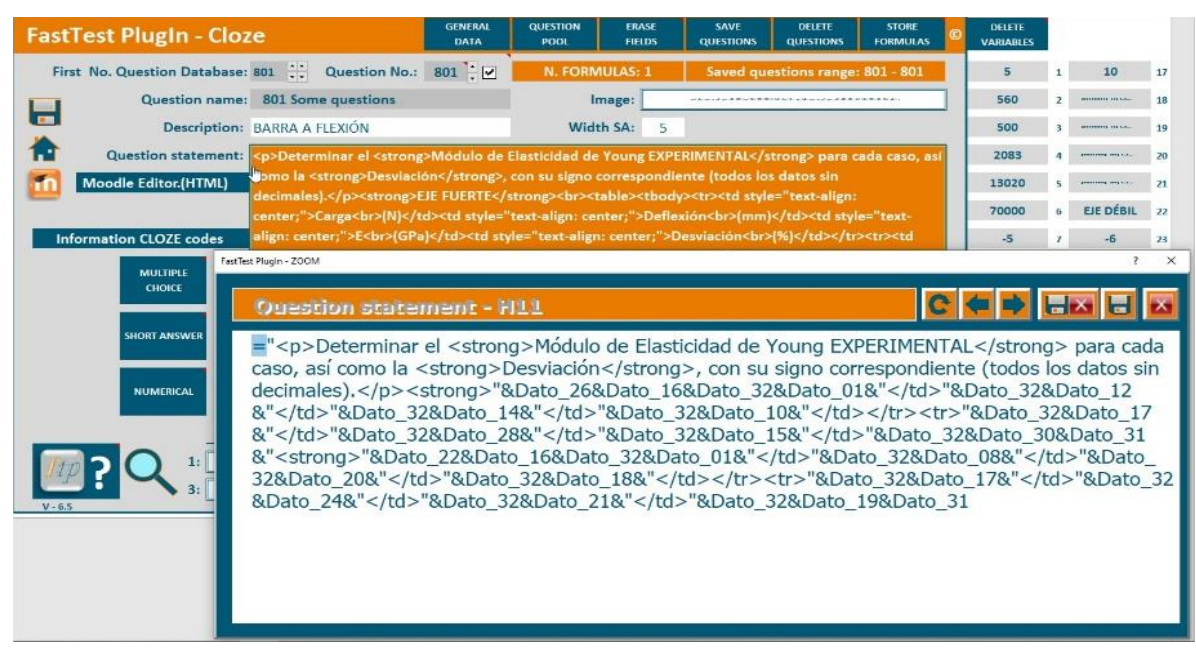

Figure 2. Example of the Zoom effect.

The number of questions of each type appears in all the screens except for the "General Data" screen, where the shown value is the one of the question types selected. In the case of parameterized questions, there is an option to "Store Formulas" to recover and reuse them. Also, this storage can be also used to save the feedbacks most commonly used.

\subsection{Fast Test PlugIn language}

Fast Test PlugIn has been developed in Spanish, and it is translated to English and French. Additionally, other languages are available (such as Italian, Portuguese and German), and Google ${ }^{\circledR}$ Translator has been used for those last three. For this reason, the "Custom Dictionary" option is made available to users that want to contribute and translate all the text of FastTest PlugIn to their native language. Another advantage of this option is that FastTest PlugIn can be translated to any language supported by Google ${ }^{\circledR}$ Translator. Figure 3.a shows the initial screen of the plugin, where the language can be selected from the left menu. Furthermore, Figure 3.b shows an example of the translation of FastTest PlugIn to a different language (Japanese in this case). In the "Custom Dictionary" screen there is a link to a Google ${ }^{\circledR}$ spreadsheet that contains the complete dictionary used in FastTest PlugIn. This spreadsheet is translated to the required language using Google ${ }^{\circledR}$ Translator and then the text is pasted in the corresponding cell of the "Custom Dictionary" screen. If any of the cells are left blank, the corresponding text will appear in the main language selected on the principal screen. 


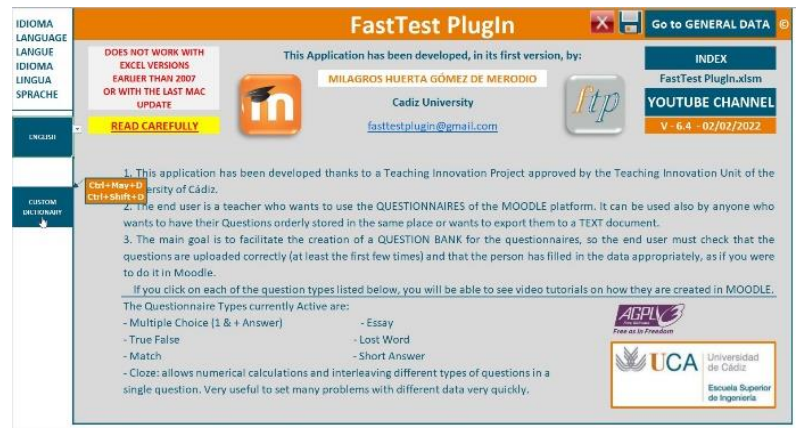

(a)

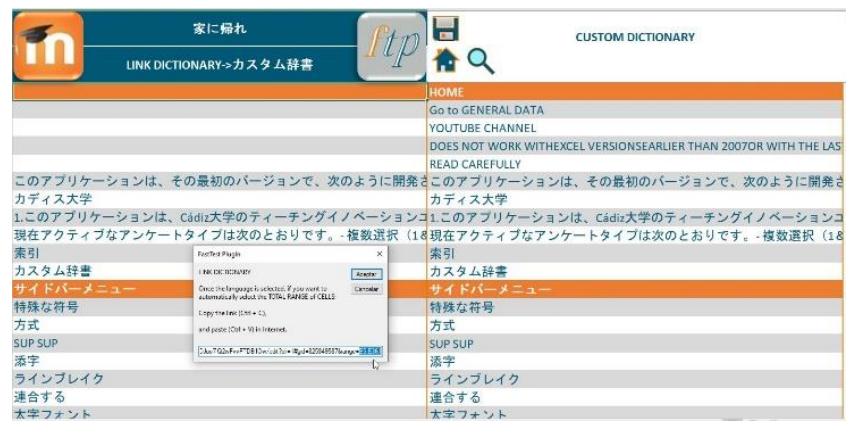

(b)

Figure 3. Language (a) selection in the initial screen and (b) custom dictionary screen.

\subsection{General Data screen}

Figure 4 shows the "General Data" screen. All the options common to the different types of questions are collected on this screen. Figure 4 also shows the drop-down menu with its different options. The main cells of the "General Data" screen are the following:

- Question type: the user selects the type of question (MCQs, true/false, matching, short answer, missing word, essay, and Cloze). Depending on the type of question, different options are displayed.

- Category and subcategory titles: they are created with their respective descriptions.

- Attempt penalty: the user indicates the penalty for multiple attempts and the question score.

- Feedback: the user can set up general feedback that appears at the end of the test and right answers. Also, setting up partially wrong answers' and wrong answers' feedback. The latter is to appear after the question.

- Question prefix: a question prefix can be configured to label the question. By doing so, all the questions related to the same topic can have different names have a common prefix.

- Initial and final number of questions to be exported: the user can select the range of questions to be exported. A warning message appears if the range selected does not contain all the questions of the spreadsheet (the message can be omitted).

- Shuffle quiz answers: the answers of the questions can be shuffled (if the question type allows it).

- Mark answers: the user can decide if the students can mark the answers in the tests. There is also an optional message that informs the students about this option.

In addition to the above, the following buttons are gathered in the right menu of the "General Data" screen (see Figure 4):

- Form: go to the form of the selected question type.

- Question Database: go to the question pool of the selected question type.

- Import from Excel Book: import previously saved questions.

- Export to Excel Book: export the selected questions to an Excel spreadsheet.

- Export to Moodle: generate an XML file with all the selected questions.

- Export to Text File: export the selected questions to a text file in order to use them in a written test.

- Export to Game: export the MCQs (single answer) to a file that can be used in a game called "game of the goose". 


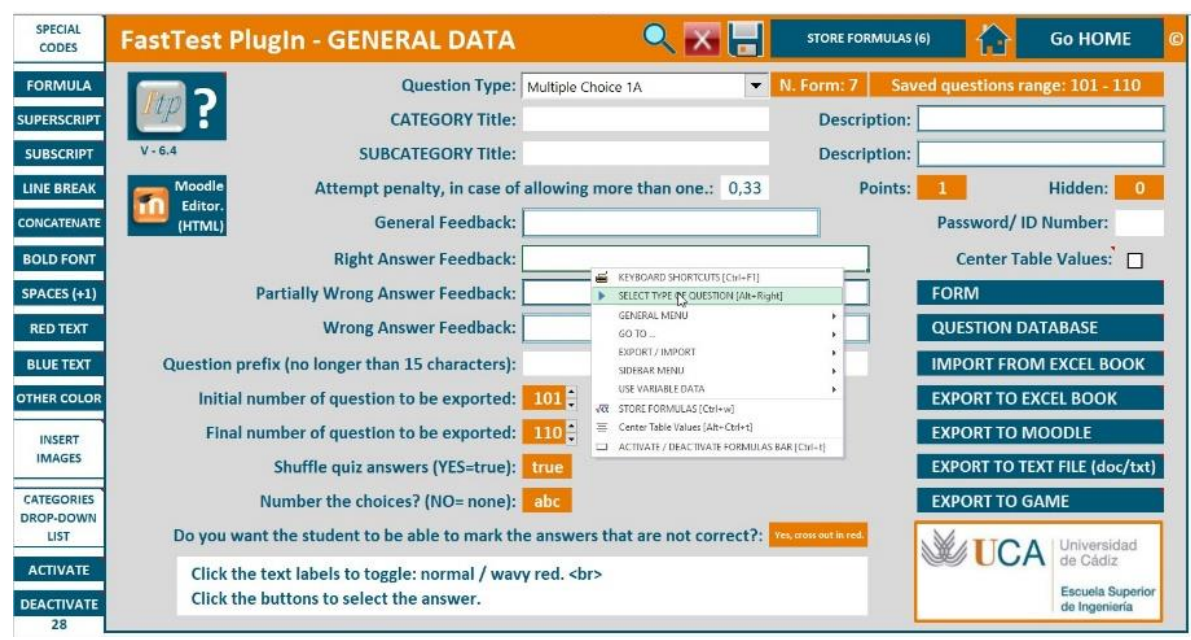

Figure 4. "General Data" screen.

\subsection{Question Database screen}

There is a question pool for each type of question, where all the question data are collected in a table (see Figure 5). The number of columns of this table depend on the input fields of each type of question. The options available in each "Question Database" screen are the same:

- General Data: goes back to the "General Data" screen.

- Form: goes to the form of the selected question type.

- Insert question: the user can introduce a question directly into the question pool (it can also be done through the form screen). Users that create questions using this option need to be familiar with the different input fields, because FastTest PlugIn does not check the entered data in this case. However, it can be a good option for the definition of questions very similar to one another, where there are few changes between the question data.

- $\quad$ Delete question: deletes the selected questions.

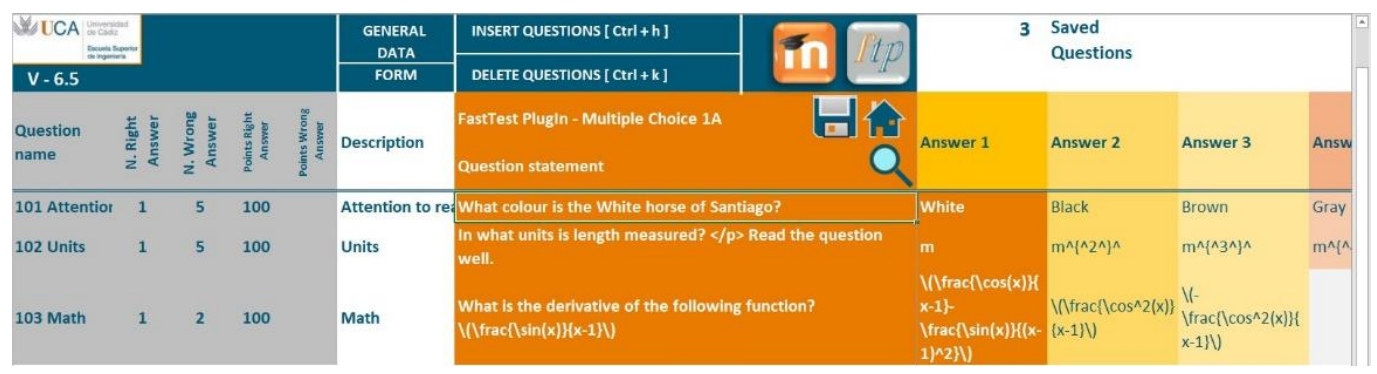

Figure 5. "Question Database" screen (which is similar to all the types of questions).

\subsection{Form screens}

As it has been stated in Section 3.3, Fast Test PlugIn support the following question types: MCQs (single and multiple answers), true/false, matching, short answer, missing word, essay, and Cloze. Each type of question has its own "Form" screen. Before going into the individual "Form" screens for each type of question, the common options are presented. In the superior menu of all the "Form" screens are the following buttons (see Figure 6):

- General Data: goes back to the "General Data" screen.

- Question Database: leads to the question pool of the selected question type.

- Erase fields: erases the content of all the cells to define a new question from scratch. This option does not delete any question from the question pool. 
- $\quad$ Save questions: saves a question in the question pool. In the case of questions created with some formulas, only the created question is saved (not the formula). If variable data are used, up to 100 questions can be saved.

- Delete questions: deletes questions one by one. If the user wants to delete a set of questions, it is easier to do it through the corresponding "Question Database" screen.

- Store formulas: saves the formulas used to create the question. It is very useful to store a feedback frequently used or to recover formulas used to create some other type of question. In addition, Fast Test PlugIn allows the importation and exportation of formulas to use them in the different versions of the plugin.

\begin{tabular}{|c|c|c|c|c|c|c|}
\hline A & $\begin{array}{l}\text { GENERAL } \\
\text { DATA }\end{array}$ & $\begin{array}{c}\text { QUESTION } \\
\text { POOL }\end{array}$ & $\begin{array}{l}\text { ERASE } \\
\text { FIELDS }\end{array}$ & $\begin{array}{c}\text { SAVE } \\
\text { QUESTIONS }\end{array}$ & $\begin{array}{c}\text { DELETE } \\
\text { QUESTIONS }\end{array}$ & $\begin{array}{c}\text { STORE } \\
\text { FORMULAS }\end{array}$ \\
\hline 1.: & $101 \div \square$ & \multicolumn{2}{|c|}{ FORMULAS: 0} & \multicolumn{3}{|c|}{ Saved questions range: 101 - 103} \\
\hline \multirow[t]{2}{*}{ ing } & & \multicolumn{2}{|c|}{ Image: } & & & \\
\hline & & \multicolumn{2}{|c|}{ Num. choices: } & & & \\
\hline
\end{tabular}

Figure 6. Superior menu of the "Form" screen.

There are also some common fields to all the "Form" screens:

- $\quad$ First Number Question Database: the user can define the first number of the question pool. Then, based on that number the questions are numbered consecutively. The name's format of the question is: Prefix - Question number - Description.

- Question number: this cell shows the number of the displayed question. Questions are visualized one by one. Question data can be changed, overwriting the previous saved data. New questions can be created based on a previous one saving the changes made with a different number of question. By doing so, the creation of similar questions is easy and fast.

- Image: the user can introduce images in the question statement. In the "Image" cell the URL of the image (with HTML syntax) should be introduced. The HTML code is easily obtained by clicking the "Insert Images" button on the left menu shown in the "Form" screen. The user has to copy-past the HTML code to the "Image" cell. This process is applicable to introduce images in all the cells with double border.

- Question statement: this cell contains the question statement. Images, formulas, variable data and HTML code can be inserted.

- Hint: the user can introduce up to four hints. In the case of tests where multiple attempts are allowed, hints can be helpful to the student. As the hint's cells have double border, HTML code is allowed. Essay is the only type of question that does not allow the definition of hints. That is because it is the only type of question that is not automatically corrected.

Now the options for each type of question are described.

\subsubsection{Multiple Choice Question Form Screen}

In the MCQ "Form" screen (see Figure 7), the possible answers are inserted in different cells. FastTest PlugIn allows the introduction of 6 possible answers. In Moodle the number of possible answers of MCQs is not limited. However, in practice it does not usually exceed 6. If more possible answers are needed, the Cloze question type can be used. Each possible answer has its corresponding mark. In this cell the user defines the score of the possible answer. In the case of MCQs with a single answer, the correct answer must have a score of 100 . The possible answer that has this score is automatically named as "right answer". And in the case of MCQs with multiple answers the sum of the scores of each right answer must be 100. If not, the total score is displayed in yellow and the question cannot be saved. It is worth mentioning that each possible answer has a feedback cell that accepts HTML code. 


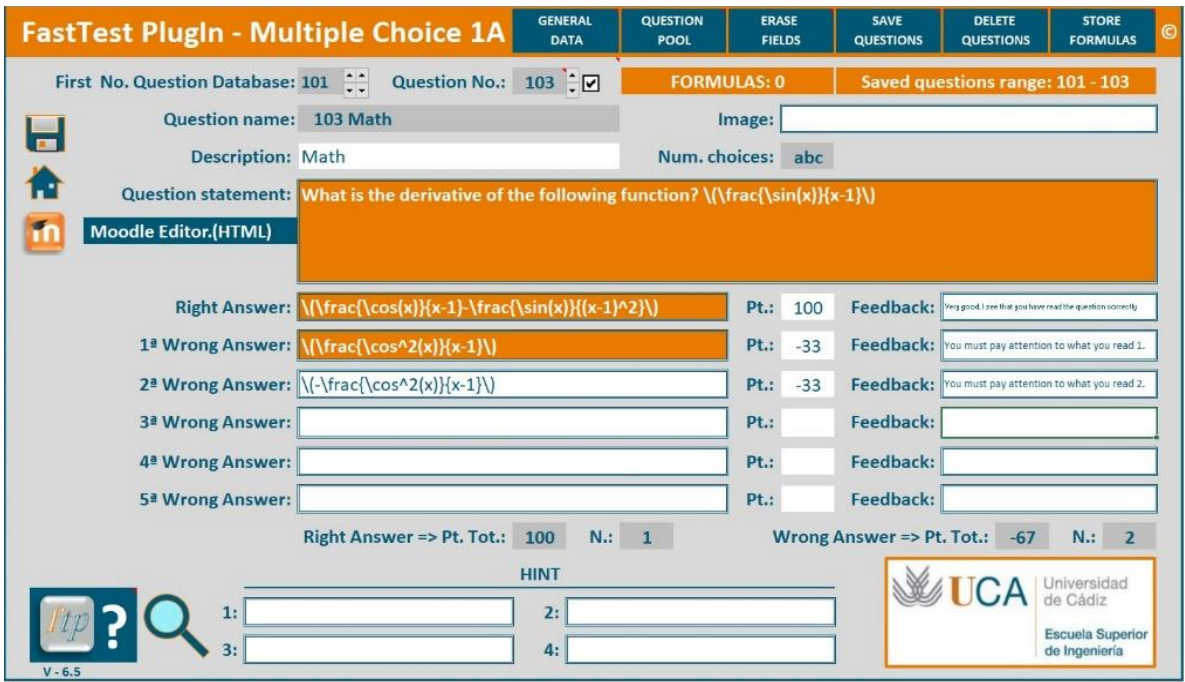

Figure 7. MCQs “Form” screen.

\subsubsection{True/False Form Screen}

For this type of question, it is necessary to indicate if the question statement is true or false. It is done in the "right answer" cell through a drop-down menu. Multiple feedbacks can be introduced for right and wrong answers (see Figure 8).

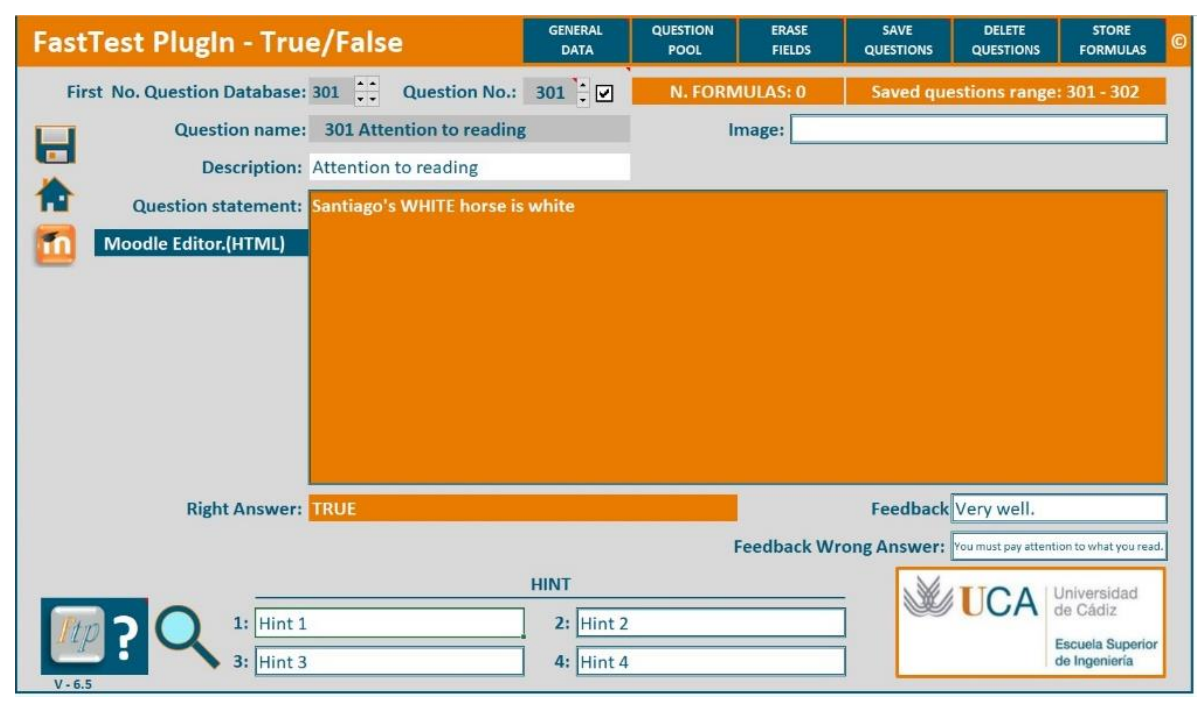

Figure 8. True/False "Form" screen.

\subsubsection{Matching Form Screen}

In the Matching "Form" screen (see Figure 9) the pairs are introduced in different cells. Fast Test PlugIn allows the introduction of 12 pairs. As in MCQs, the number of pairs is not limited in Moodle. However, it is considered that 12 pairs are fully adequate for practical tests. The members of the pairs are grouped in two groups. In group 1 the user has to introduce the member of the pair that will be shown in the test. HTML code is accepted in group 1 cells. Besides, in group 2 the user has to introduce the other member of the pair corresponding to the correct answer. The cells of group 2 can only contain plain text because the student will select the answer from a drop-down menu. 


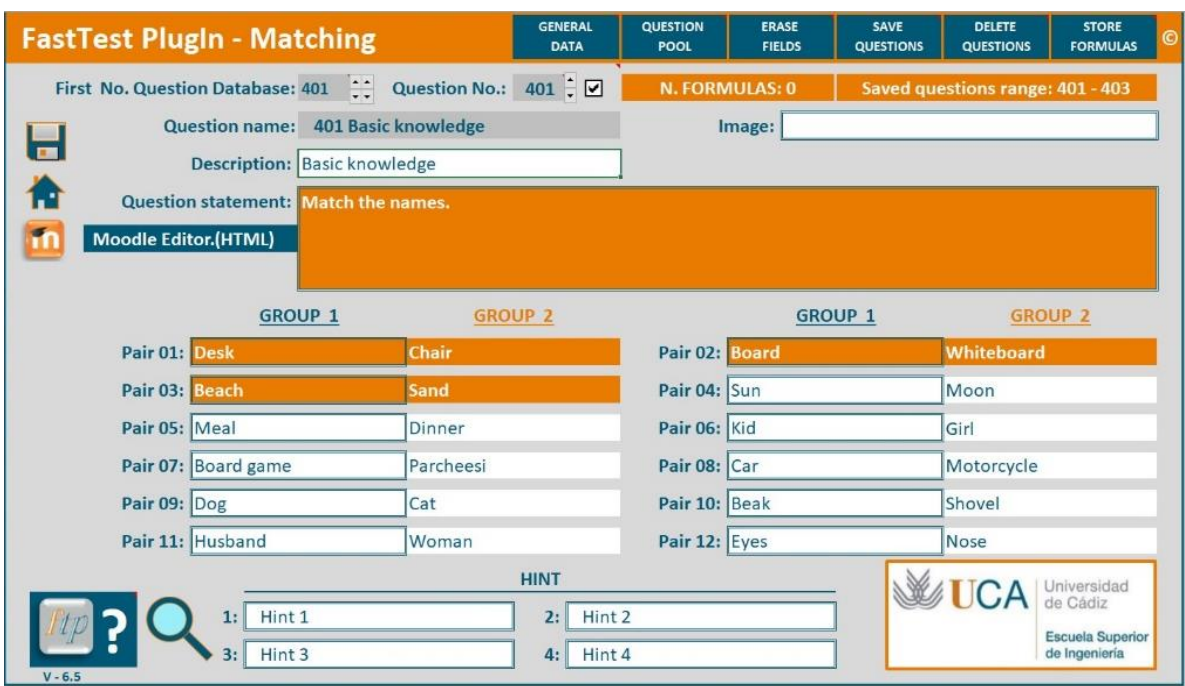

Figure 9. Matching "Form" screen.

\subsubsection{Short Answer Form Screen}

First of all, the case sensitivity of the answers introduced by the students has to be defined. There are 8 possible answer cells (see Figure 10). As in the previous cases, this number has been defined based on experience. Cloze questions can be used if more answers are needed. Each answer has a score cell, and there can be answers with different scores. Finally, each answer has a feedback cell that accepts HTML code.

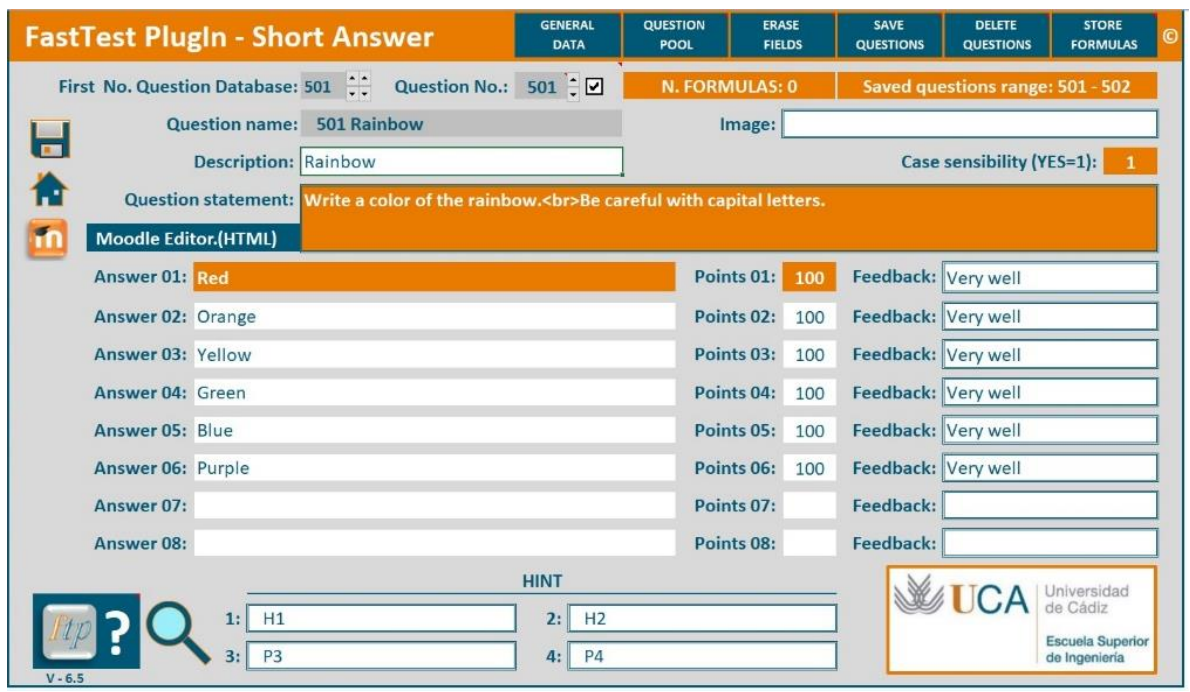

Figure 10. Short Answer "Form" screen.

\subsubsection{Missing Word Form Screen}

The definition of Missing Word questions is not very intuitive in Moodle. Fast Test PlugIn eases the process of defining Missing Word questions. In this plugin 12 missing words are allowed (see Figure 11). The chosen maximum number is based on practice. All the words are grouped by the user. The words that belong to the same group will appear as possible answers in the same drop-down menu. The cells named "Word" contains the answers and the "N. Group" cells contain the number of the group. In the question statement, some words (the missing ones) are replaced by the number of a group of words between double square brackets. This group of words contains the possible answers, they are shown in a drop-down menu. 


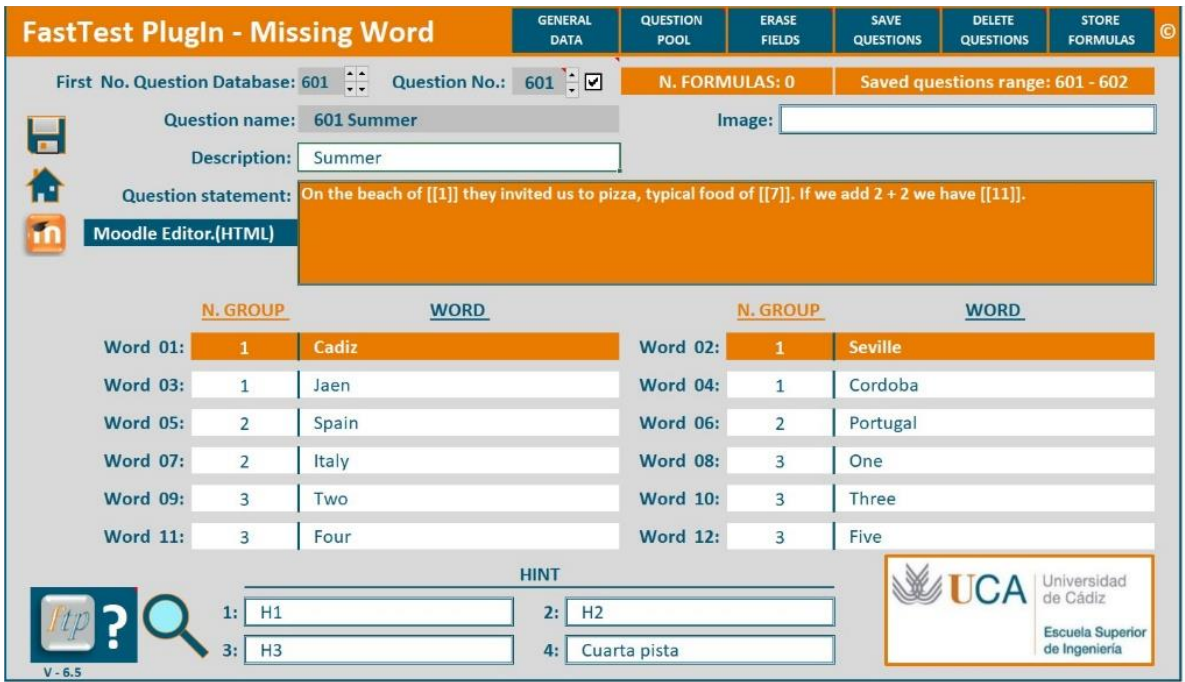

Figure 11. Missing Word "Form" screen.

\subsubsection{Essay Form Screen}

In the Essay "Form" screen (see Figure 12) the fields "Response format", "Require text", "Input box size", "Allow attachments" and "Require attachments" are drop-down menus that shows the same options that Moodle allows for this type of question. There is also two optional fields. One of them is "Response template", where the user can introduce a question answer template for students. The other is "Information for the evaluator" that contains the criterion for the evaluation of the question.

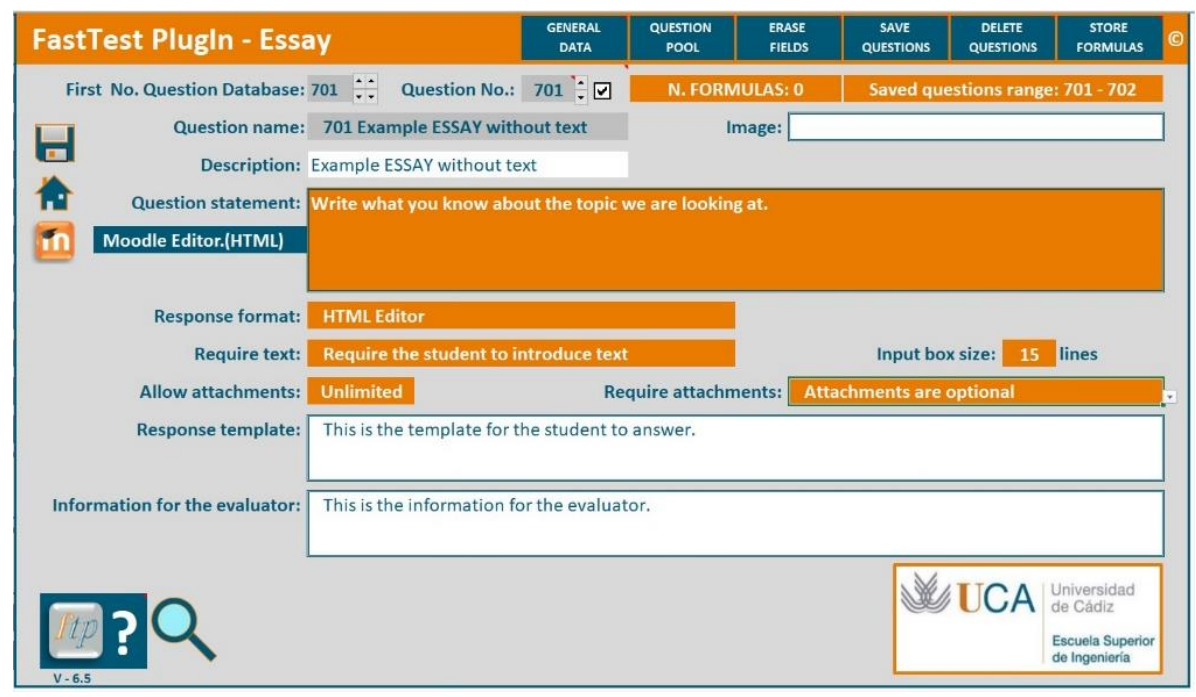

Figure 12. Essay “Form” screen.

\subsubsection{Cloze Form Screen}

The main cell of the Cloze "Form" screen (see Figure 13) is the "Question statement" cell. Cloze questions require a special syntax. The Cloze "Form" screen shows different buttons which help the user during the introduction of the question statement. In addition, there is a link to the Moodle's Editor, where the question statement code can be defined without great expertise. The user can define the width of the answer text boxes in the "Width SA" cell. When a Cloze question is defined in Moodle, the width of the answer text box is adapted to the length of the correct answer. By defining a common width, all the answer text boxes will have the same width so that students do not have extra clues about the length of the answer. 


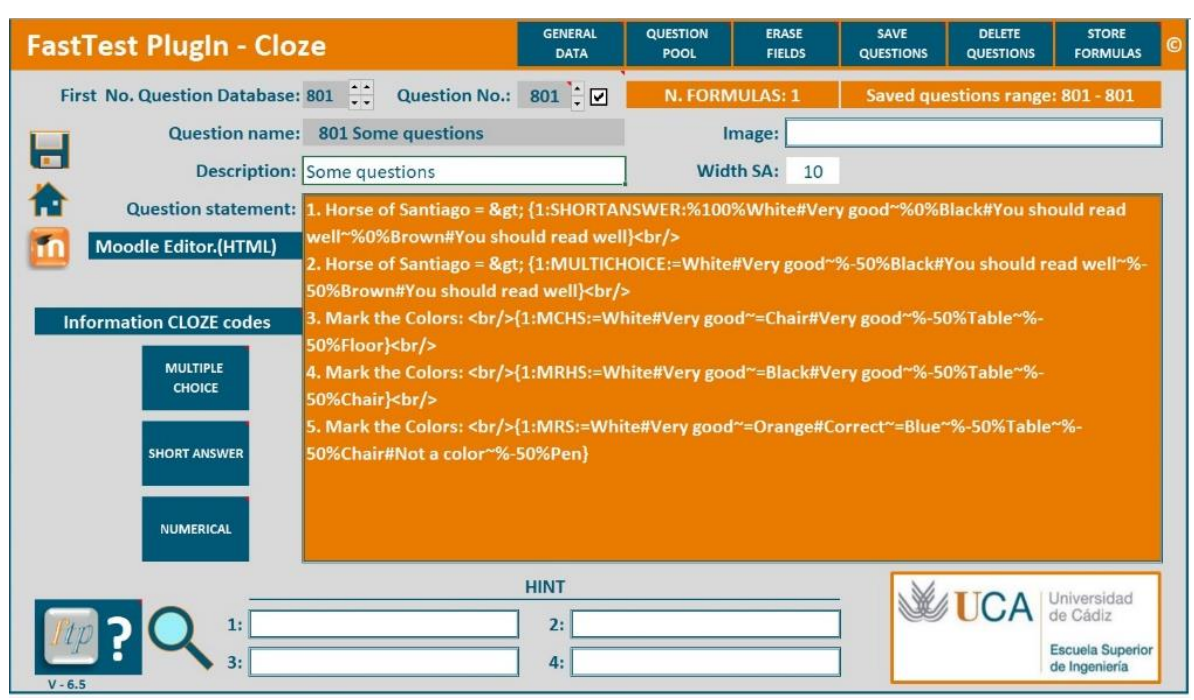

Figure 13. Cloze "Form" screen

\section{Results and Discussion}

In this Section, the most important plugins to import/export questions into Moodle are compared. These plugins have been selected from the extensive literature review carried out in Section 2. From the web-based plugins described in Section 2.2.1, VLEEtools.com has been selected because it supports a larger number of different question types. Moodle2Word has been selected between the plugins developed in text processors described in Section 2.2.3 due to its widespread use in the Moodle's community. Question Machine has been selected from the specialized software plugins defined in Section 2.2.4 because it supports a larger number of different question types and it is oriented to Moodle's users. Finally, FastTest PlugIn is selected between the spreadsheets plugins defined in Section 2.2.2 due to its high functionality.

Table 1 shows the question types supported by each plugin. FastTest PlugIn, Moodle2Word and VLEtools.com has implemented a high number of different question types. It should be pointed out that numerical questions can be introduced in FastTest PlugIn through a Cloze question. Also, description questions simply shows some text (and possibly images too) without requiring any answer (they are more labels than questions). For this reason, this type of questions is not considered as relevant as the rest. One of the main advantages of FastTest PlugIn, because it is based on an Excel spreadsheet, is that parameterized questions can be created easily.

Table 1. Question types comparison.

\begin{tabular}{lcccc}
\hline Question type & $\begin{array}{c}\text { VLEtools } \\
\text {.com }\end{array}$ & $\begin{array}{c}\text { Moodle2 } \\
\text { Word }\end{array}$ & $\begin{array}{c}\text { Question } \\
\text { Machine }\end{array}$ & $\begin{array}{c}\text { FastTest } \\
\text { PlugIn }\end{array}$ \\
\hline MCQ & $\mathrm{X}$ & $\mathrm{X}$ & $\mathrm{X}$ & $\mathrm{X}$ \\
True / False & $\mathrm{X}$ & $\mathrm{X}$ & $\mathrm{X}$ & $\mathrm{X}$ \\
Matching & $\mathrm{X}$ & $\mathrm{X}$ & $\mathrm{X}$ & $\mathrm{X}$ \\
Missing Word & & $\mathrm{X}$ & & $\mathrm{X}$ \\
Essay & $\mathrm{X}$ & $\mathrm{X}$ & & $\mathrm{X}$ \\
Short Answer & $\mathrm{X}$ & $\mathrm{X}$ & $\mathrm{X}$ & $\mathrm{X}$ \\
Cloze & $\mathrm{X}$ & $\mathrm{X}$ & $\mathrm{X}$ & $\mathrm{X}$ \\
Numerical & $\mathrm{X}$ & $\mathrm{X}$ & $\mathrm{X}$ & \\
Calculated & & & & $\mathrm{X}$ \\
Description & $\mathrm{X}$ & $\mathrm{X}$ & & \\
\hline
\end{tabular}

${ }^{1}$ This type of questions is not directly considered in FastTest PlugIn, but it can be created with Excel functions. 
Table 2 shows the available languages of the four studied plugins. FastTest PlugIn stands above the rest because it not only can include all the languages supported by Google Translator, but also the user can personalize the text shown in all the buttons and drop-down menus.

Table 2. Available languages comparison.

\begin{tabular}{lcccc}
\hline Language & $\begin{array}{c}\text { VLEtools } \\
\text {.com }\end{array}$ & $\begin{array}{c}\text { Moodle2 } \\
\text { Word }\end{array}$ & $\begin{array}{c}\text { Question } \\
\text { Machine }\end{array}$ & $\begin{array}{c}\text { FastTest } \\
\text { PlugIn }\end{array}$ \\
\hline English & $\mathrm{X}$ & $\mathrm{X}$ & $\mathrm{X}$ & $\mathrm{X}$ \\
Spanish & $\mathrm{X}$ & $\mathrm{X}$ & & $\mathrm{X}$ \\
French & $\mathrm{X}$ & $\mathrm{X}$ & & $\mathrm{X}$ \\
Portuguese & $\mathrm{X}$ & & & $\mathrm{X}$ \\
Italian & $\mathrm{X}$ & & & $\mathrm{X}$ \\
Dutch & $\mathrm{X}$ & & & $\mathrm{X}$ \\
Google Translator & $\mathrm{X}$ & & & $\mathrm{X}$ \\
\hline
\end{tabular}

Table 3 shows the comparison of other important characteristics of the studied plugins. Moodle2Word is the only plugin that needs to be installed in Moodle. This can be an inconvenience because only a Moodle administrator can install this type of plugins. The rest of the studied plugins do not require installation in Moodle. With regard to the operating system, Moodle2Word, Question Machine and FastTest PlugIn are compatible with Microsoft ${ }^{\circledR}$ Windows while VLEtools.com is compatible with all the operating systems. FastTest PlugIn is not fully compatible with macOS (depends on its version and not all the functionalities are available); a Question Machine version compatible with macOs is under development.

It needs to be highlighted that FastTest PlugIn is the only plugin that allows the creation of parameterized questions. FastTest PlugIn and Moodle2Word require commercial software for its use (Microsoft ${ }^{\circledR}$ Excel and Word respectively), while VLEtools.com and Question Machine do not. Due to the widespread use of the Microsoft ${ }^{\circledR}$ Office package over the world, its requirement is not considered as a major drawback. FastTest PlugIn, Moodle2Word and Question Machine allow the exportation of the questions into text files to make written tests. Talking about gamification, the only plugin that allows the creation of games based on the question pool is FastTest PlugIn with the "game of the goose". Finally, FastTest PlugIn, Moodle2Word and Question Machine allow the introduction of images. Moreover, FastTest PlugIn allows the introduction of images found on the Internet using an URL in any of the fields that allow HTML code. In addition, another plugin has been prepared, ForImage DataBase [78], which allows the creation of a bank of images and formulas.

Table 3. Comparison of other characteristics.

\begin{tabular}{|c|c|c|c|c|}
\hline Other characteristics & $\begin{array}{l}\text { VLEtools } \\
\text {.com }\end{array}$ & $\begin{array}{c}\text { Moodle2 } \\
\text { Word }\end{array}$ & $\begin{array}{l}\text { Question } \\
\text { Machine }\end{array}$ & $\begin{array}{c}\text { FastTest } \\
\text { PlugIn }\end{array}$ \\
\hline Installation in Moodle & $\mathrm{NO}$ & YES & $\mathrm{NO}$ & $\mathrm{NO}$ \\
\hline Operating system & All & Windows & Windows $^{1}$ & $\begin{array}{c}\text { Windows / } \\
\text { macOS }^{2}\end{array}$ \\
\hline Parameterized questions & NO & $\mathrm{NO}$ & NO & YES \\
\hline Require comercial software & $\mathrm{NO}$ & YES & NO & YES \\
\hline Written tests & $\mathrm{NO}$ & YES & YES & YES \\
\hline Gamification & $\mathrm{NO}$ & NO & $\mathrm{NO}$ & YES \\
\hline Images & $\mathrm{NO}$ & YES & YES & YES \\
\hline
\end{tabular}

${ }^{1}$ A version compatible with macOs is under development.

${ }^{2}$ FastTest PlugIn is not compatible with the last version of macOs; and in previous versions not all the functionalities of the plugin are available 
FastTest PlugIn and Moodle2Word are the most complete plugins to export questions to Moodle. Both require additional commercial software, but FastTest PlugIn does not require installation in Moodle. In addition, as FastTest PlugIn is based on an Excel spreadsheet, it has all the advantages of this commercial software in terms of calculation power. Parameterized questions can be defined based on all the functions of Excel, which is a great advantage that FstTest PlugIn has. Finally, FastTest PlugIn's questions can be grouped by topics, difficulty, subjects, etc.

\section{Conclusions}

In this piece of work, a new plugin to export questions to Moodle has been presented: FastTest PlugIn.

First of all, an extensive literature review is carried out. Then, the most important plugins selected from the literature review are described more in detail. Plugins are grouped by the computing resources needed.

FastTest PlugIn allows the definition of MCQ, true/false questions, matching questions, short answer questions, essay questions, missing words questions, and Cloze questions in an easy and intuitive way. As FastTest PlugIn is based on a Microsoft ${ }^{\circledR}$ Excel spreadsheet, it also helps with the creation of the question. Since Excel functions can be used to compute the results in numerical questions, faculty members will only have to introduce the corresponding formulas and the result will be calculated automatically. By doing so, a decrease in the number of mistakes made during the question data introduction is observed. In addition, the use of Excel functions allows the definition of parameterized questions.

Gamification of education is a strategy to increase the student's engagement by the use of typical elements of game playing (e.g. point scoring and competition with others) in an educational environment. FastTest PlugIn allows the definition of gamified versions of traditional tests through the "game of the goose" option. Finally, FastTest PlugIn includes all the languages supported by Google Translator.

Four plugins have been compared: FastTest PlugIn, VLEtools.com, Moodle2Word, and Question Machine. It can be concluded that FastTest PlugIn and Moodle2Word are the most complete plugins to export questions to Moodle. And it has been shown that FastTest PlugIn has some advantages in comparison with Moodle2Word such as its higher calculation power (because it is based on an Excel spreadsheet), gamification possibilities and the possibility to create parameterized questions.

\section{Patents}

FastTest PlugIn has been registered as "Computer Program" in Spain (reference 04/2021/4745).

Author Contributions: M.H.: Conceptualization, software, validation, investigation, writing - original draft preparation. M.A.F.R.: visualization, writing - original draft preparation.

Acknowledgments: the present piece of work was partly financed by the Unidad de Formación e Innovación Docente of the University of Cádiz in the form of a "Proyecto de Innovación y Mejora Docente, code "sol-202000162327-tra" and title "Elaboración de grandes bancos de preguntas/problemas, como complemento a la docencia digital.". This support is gratefully acknowledged.

Conflicts of Interest: The authors declare no conflict of interest.

\section{References}

1. Cuaton, G. Philippines higher education institutions in the time of COVID-19 pandemic. evista Rom. Pentru Educ. Multi- Dimens. 2020, 12, 61-70.

2. Wang, C.; Cheng, Z.; Yue, X.G. Risk management of COVID-19 by universities in China. J. Risk Financ. Manag. 2020, 13, 36.

3. Villegas-Ch, W.; Palacios-Pacheco, X.; Roman-Cañizares, M.; Luján-Mora, S. Analysis of educational data in the current state of 
university learning for the transition to a hybrid education model. Appl. Sci. 2021, 11, 1-19, doi:10.3390/app11052068.

4. Bisht, R.K.; Jasola, S.; Bisht, I.P. Acceptability and challenges of online higher education in the era of COVID-19: a study of students' perspective. Asian Educ. Dev. Stud. 2020, doi:https://doi.org/10.1108/AEDS-05-2020-0119.

5. Kummitha, H.R.; Kolloju, N.; Chittoor, P.; Madepalli, V. Coronavirus disease 2019 and its effect on teaching and learning process in the higher educational institutions. High. Educ. Futur. 2021, 8, 90-107.

6. Pokhrel, S.; Chhetri, R. A literature review on impact of COVID-19 pandemic on teaching and learning. High. Educ. Futur. 2021, 8, 133-141.

7. Sahu, K.K.; Mishra, A.K.; Lal, A. Coronavirus disease-2019: An update on third coronavirus outbreak of 21st century. QJM An Int. J. Med. 2020, 113, 384-386.

8. Chhetri, B.; Goyal, L.M.; Mittal, M.; Battineni, G. Estimating the prevalence of stress among Indian students during the COVID19 pandemic: A cross-sectional study from India. Med. Sci. 2021, 16, 260-267.

9. Son, C.; Hegde, S.; Smith, A.; Wang, X.; Sasangohar, F. Effects of COVID-19 on college Students' mental health in the United States: Interview survey study. J. Med. Internet Res. 2020, 22.

10. Aoki, K.; Pogroszewski, D. Virtual University Reference Model: A Guide to Delivering Education and Support Services to the Distance Learner. Online J. Distance Learn. Adm. 1998, 1, 16.

11. Cloete, E. Electronic education system model. Comput. Educ. 2001, 36, 171-182, doi:10.1016/S0360-1315(00)00058-0.

12. Feidakis, M.; Daradoumis, T.; Caballé, S.; Conesa, J. Embedding emotion awareness into e-learning environments. Int. J. Emerg. Technol. Learn. 2014, 9, 39-46, doi:10.3991/ijet.v9i7.3727.

13. Dahlstrom, E.; Brooks, D.C.; Bichsel, J. The current ecosystem of learning management systems in higher education: Student, faculty, and IT perspectives; Research report. Louisville, CO: ECAR, 2014;

14. Gil, I. Educomunicación ¿Online?: actuación del profesorado universitario ante los escenarios de la crisis de la COVID-19. In La comunicación especializada del Siglo XXI; McGraw-Hill, 2020.

15. Crawford, J.; Butler-Henderson, K.; Rudolph, J.; Malkawi, B.; Glowatz, M.; Burton, R.; Magni, P.; Lam, S. COVID-19: 20 countries' higher education intra-period digital pedagogy responses. J. Appl. Teach. Learn. 2020, 3, 9-28.

16. Ilgaz, H.; Adanir, G.A. Providing online exams for online learners: Does it really matter for them. Educ. Inf. Technol. 2020, 25, 1255-1269.

17. Shakeel, A.; Shazli, T.; Salman, M.S.; Naqvi, H.R.; Ahmad, N.; Ali, N. Challenges of unrestricted assignment-based examinations (ABE) and restricted open-book examinations (OBE) during COVID-19 pandemic in India: An experimental comparison. Hum. Behav Emerg Tech 2021, 3, 1050-1066, doi:10.1002/hbe2.290.

18. Antón-Sancho, Á.; Vergara, D.; Lamas-álvarez, V.E.; Fernández-Arias, P. Digital content creation tools: American university teachers' perception. Appl. Sci. 2021, 11, doi:10.3390/app112411649.

19. López-Zambrano, J.; Lara, J.A.; Romero, C. Towards portability of models for predicting students' final performance in university courses starting from moodle logs. Appl. Sci. 2020, 10, doi:10.3390/app10010354.

20. Bayazit, A.; Askar, P. Performance and duration differences between online and paper-pencil test. Asia Pacific Educ. Rev. 2012, 13, 219-226.

21. Parshall, C.G.; Spray, J.A.; Kalohn, J.C.; Davey, T. Practical considerations in computer-based testing; Springer, 2002;

22. Randy, G. E-learning in the 21st century. A Framew. Res. Pract. 2011, 2, 110-111.

23. Farooq, F.; Rathore, F.A.; Mansoor, S.N. Challenges of online medical education in Pakistan during COVID-19 pandemic. J. Coll. Physicians Surg. Pakistan 2020, 30, 67-69.

24. Qazi, A.; Naseer, K.; Qazi, J.; AlSalman, H.; Naseem, U.; Yang, S.; Hardaker, G.; Gumaei, A. Conventional to online education during COVID-19 pandemic: Do develop and underdeveloped nations cope alike. Child. Youth Serv. Rev. 2020, 119, 105582.

25. Xie, X.; Siau, K.; Nah, F.F. COVID-19 pandemic: Online education in the new normal and the next normal. J. Inf. Technol. Case Appl. Res. 2020, 22, 175-187. 
26. Fask, A.; Englander, F.; Wang, Z. Do online exams facilitate cheating? An experiment designed to separate possible cheating from the effect of the online test taking environment. J. Acad. Ethics 2014, 12, 101-112.

27. Mellar, H.; Peytcheva-Forsyth, R.; Kocdar, S.; Karadeniz, A.; Yovkova, B. Addressing cheating in e-assessment using student authentication and authorship checking systems: teachers' perspectives. International , 14, 2. J. Educ. Integr. $2018,14$.

28. Sureda Negre, J.; Comas Forgas, R.; Gili Planas, M. Prácticas académicas deshonestas en el desarrollo de exámenes entre el alumnado universitario español. Estud. Sobre Educ. 2009, 103-122.

29. Comas, R.; Sureda, J.; Casero, A.; Morey, M. La integridad académica entre el alumnado universitario Español. Estud. Pedagog. 2011, 37, 207-225, doi:10.4067/S0718-07052011000100011.

30. Rowe, N. Cheating in online student assessment: Beyond plagiarism. Online J. Distance Learn. Adm. 2004, 7, 1-10.

31. Huerta, M.; Portela, J.M.; Pastor, A.; Otero, M.; Velázquez, S.; González, P. Cómo preparar una gran colección de problemas virtuales, para que los alumnos aprendan. In Proceedings of the VIII Jornadas Internacionales de Innovación Universitaria; Cádiz, 2011; pp. 1-7.

32. Montejo Bernardo, J.M. Exámenes no presenciales en época del COVID-19 y el temor al engaño. Un estudio de caso en la Universidad de Oviedo. Magister 2020, 32, 102-110, doi:10.17811/msg.32.1.2020.102-110.

33. Shakeel, A.; Shazli, T.; Salman, M.S.; Naqvi, H.R.; Ahmad, N.; Ali, N. Challenges of unrestricted assignment-based examinations $(\mathrm{ABE})$ and restricted open-book examinations (OBE) during COVID-19 pandemic in India: An experimental comparison. Hum. Behav Emerg Tech 2021, 1050-1066, doi:10.1002/hbe2.290.

34. Haus, G.; Pasquinelli, Y.B.; Scaccia, D.; Scarabottolo, N. Online written exams during COVID-19 crisis. Proc. 14th IADIS Int. Conf. e-Learning 2020, EL 2020 - Part 14th Multi Conf. Comput. Sci. Inf. Syst. MCCSIS 2020 2020, 79-86, doi:10.33965/el2020_2020071010.

35. Wahid, R.; Farooq, O. Online Exams in the Time of COVID-19: Quality Parameters. Int. J. Soc. Sci. \E Educ. Stud. 2020, 7, 13-21, doi:10.23918/ijsses.v7i4p13.

36. Boitshwarelo, B.; Reedy, A.K.; Billany, T. Envisioning the use of online tests in assessing twenty-first century learning: a literature review. Res. Pract. Technol. Enhanc. Learn. 2017, 12, 16, doi:10.1186/s41039-017-0055-7.

37. Ahmed, F.R.A.; Ahmed, T.E.; Saeed, R.A.; Alhumyani, H.; Khalek, S.A.; Zinadah, H.A. Analysis and challenges of robust Eexams performance under COVID-19. Results Phys. 2021, 23, 103987.

38. Lee, S.; Choi, Y.J.; Kim, H.S. The accurate measurement of students' learning in e-learning environments. Appl. Sci. 2021, 11, doi:10.3390/app11219946.

39. Stone, D.E.; Zheng, G. Learning management systems in a changing environment. In Handbook of research on education and technology in a changing society; Hershey: IGI Global, 2014; pp. 756-767.

40. Gipps, C. V. What is the role for ICT-based assessment in universities? Stud. High. Educ. 2005, 30, 171-180.

41. Brady, A.M. Assessment of learning with multiple-choice questions. Nurse Educ. Pract. 2005, 5, $238-242$.

42. Donnelly, C. The use of case based multiple choice questions for assessing large group teaching: Implications on student's learning. Irish J. Acad. Pract. 2014, 3, 12.

43. Baleni, Z.G. Online formative assessment in higher education: its pros and cons. Electron. J. e-Learning 2015, 13, $228-236$.

44. Douglas, M.; Wilson, J.; Ennis, S. Multiple-choice question tests: a convenient, flexible and effective learning tool? A case study. Innov. Educ. Teach. Int. 2012, 49, 111-121.

45. Harmon, O.R.; Lambrinos, J.; Buffolino, J. Assessment design and cheating risk in online instruction. Online J. Distance Learn. Adm. 2010, 13.

46. Gamage, S.H.P.W.; Ayres, J.R.; Behrend, M.B. A systematic review on trends in using Moodle for teaching and learning. Int. J. STEM Educ. 2022, 9, 9.

47. Sáiz-Manzanares, M.C.; Rodríguez-Díez, J.J.; Díez-Pastor, J.F.; Rodríguez-Arribas, S.; Marticorena-Sánchez, R.; Ji, Y.P. Monitoring of student learning in learning management systems: An application of educational data mining techniques. Appl. Sci. 2021, 11, doi:10.3390/app11062677. 
48. Setiadi, P.M.; Alia, D.; Sumardi, S.; Respati, R.; Nur, L. Synchronous or asynchronous? Various online learning platforms studied in Indonesia 2015-2020. J. Phys. Conf. Ser. 2021, 1987.

49. Shkoukani, M. Explore the major characteristics of learning management systems and their impact on e-learning success. Int. J. Adv. Comput. Sci. Appl. 2019, 10, 296-301.

50. Xin, N.S.; Shibghatullah, A.S.; Subaramaniam, K.A.P.; Wahab, M.H.A. A systematic review for online learning management system. J. Phys. Conf. Ser. 2021, 1874, 12030.

51. Altinpulluk, H.; Kesim, M. A systematic review of the tendencies in the use of learning management systems. Turkish Online J. Distance Educ. 2021, 22, 40-54.

52. Sergis, S.; Vlachopoulos, P.; Sampson, D.G.; Pelliccione, L. Implementing teaching model templates for supporting fipped classroomenhanced STEM education in Moodle. In Handbook on Digital Learning for K-12 Schools; Marcus-Quinn, A., Hourigan, T., Eds.; Springer International Publishing, 2017; pp. 191-215.

53. Moodle Moodle statistics; https://stats.moodle.org/. Accesed 4 Feb 2022;

54. Moodle Moodle plugins; https://moodle.org/plugins/. Accesed 4 Feb 2022;

55. Svien, J. Streamlining Moodle's Question Creation Process with Excel. In Proceedings of the Proceedings of MoodleMoot Japan 2017 Annual Conference; 2017; pp. 34-39.

56. Mintii, I.S.; Shokaliuk, S. V.; A.Vakaliuk, T.; Mintii, M.M.; Soloviev, V.N. Import test questions into Moodle LMS; 2019;

57. Moodle Moodle Plugins; https://moodle.org/plugins/. Accesed 15 Feb 2022;

58. Huerta, M. FastTest PlugIn Available online: https://moodle.org/plugins/view.php?id=2831.

59. Moodle Import questions; https://docs.moodle.org/311/en/Import_questions. Accesed 15 Feb 2022;

60. Moodle Question formats statistics; https://moodle.org/plugins/stats.php?plugin_category=30. Accesed 15 Feb 2022;

61. Moodle Utilities statistics; https://moodle.org/plugins/stats.php?plugin_category=59. Accesed 15 Feb 2022;

62. Alonso, F.; Garzón, V.; Jiménez, J.A.; Arbeláez, R.F. Generation of Random Questions with Multiple Choice Single Answer for Moodle. Lámpsakos 2011, 3, 32-37.

63. Hendrickson, A.; College, S.N.; Pere, D. The moodle package : generating Moodle quizzes via LaTeX 2016, 1-12.

64. Ivanova, Y. Vletools: Moodle XML Converter; http://www.vletools.com/converter/quiz. Accesed 15 Feb 2022;

65. Vilas, M. Moodle test creator; http://text2gift.atwebpages.com/Text2GiftConverter.html. Accesed 15 Feb 2022;

66. Bollaert, H. CQ4M Generator; https://sites.google.com/site/cq4mgenerator/home. Accesed 15 Feb 2022;

67. González Gijón, V. Herramienta basada en VBA para generación de bancos de preguntas en Moodle 2021.

68. Hata, A.; Ueki, S.; Toyama, K. Moodle 小テスト問題, アンケート質問の一括作成ッール; https://www.itc.utoyama.ac.jp/moodle3/tool/. Accesed 15 Feb 2022, 2020;

69. Hata, A.; Ueki, S.; Toyama, K. Improvement of Word-XML Conversion Tools and Expansion of Launcher -. In Proceedings of the Proceedings of MoodleMoot Japan 2019 Annual Conference; 2019; pp. 1-48.

70. Libre Gift; https://code.google.com/archive/p/libre-gift/. Accesed 15 Feb 2022;

71. Campbell, E. Microsoft Word File Import/Export (Question Format); https://moodle.org/plugins/qformat_wordtable. Accesed 15 Feb 2022;

72. Question Machine 2012.

73. Gourdie, J.; Fendall, A. Question machine: A tool for creating online assessments in the blended learning and Moodle environment. In Proceedings of the New Zealand Applied Business Education Conference 2012: Enhance, Engage, Educate; Hamilton, New Zealand., 2012; Vol. 43, pp. 325-443.

74. Galli, L. QuizFaber; https://www.quizfaber.com/index.php/en/. Accesed 15 Feb 2022, 2021;

75. Huerta, M.; Fernández Ruiz, M.A. FTP: FastTest PlugIn, aplication to create big question banks of different types for the Moodle platform. In Proceedings of the CINAIC 2021: VI Congreso Internacional sobre Aprendizaje, Innovación y Cooperación; 2021; pp. 643-648. 
76. Huerta, M. Herramienta para crear de manera rápida Bancos de Preguntas para los Cuestionarios de MOODLE Available online: https://www.youtube.com/watch?v=gISZK119IL8.

77. Huerta, M. GitHub Release Stats - FastTest-PlugIn; https://tooomm.github.io/github-releasestats/?username=MilagrosHuerta\&repository=FastTest-PlugIn. Accesed 15 Feb 2022, 2021.

78. Huerta, M. ForImage DataBase; Available online: https:/github.com/MilagrosHuerta/ForImageDatabase/releases/download/V2.0/ForImage.Database.zip. 